\title{
A new 21-cm absorber identified with an $L \sim L^{\star}$ galaxy
}

\author{
N. Kanekar ${ }^{1}$, R. M. Athreya ${ }^{2}$, and J. N. Chengalur ${ }^{1}$ \\ 1 National Centre for Radio Astrophysics, Post Bag 3, Ganeshkhind, Pune 411 007, India \\ e-mail: chengalu@ncra.tifr.res.in \\ 2 European Southern Observatory, Alonso de Cordova 3107, Vitacura, Santiago 19, Chile \\ e-mail: rathreya@eso.org
}

Received 26 October 2001 / Accepted 20 November 2001

\begin{abstract}
We present Giant Metrewave Radio Telescope (GMRT) observations of redshifted 21-cm absorption from the $z=0.437$ metal line absorption system towards PKS 1243-072. HI absorption is clearly detected; the absorption profile has a velocity spread of $\sim 20 \mathrm{~km} \mathrm{~s}^{-1}$. Detection of 21-cm absorption indicates that the absorber has an HI column density large enough to be classified as a damped Lyman- $\alpha$ system. Follow up ground based optical imaging and spectroscopy allow us to identify the absorber with an $L \sim L^{\star}$ galaxy at an impact parameter of $\sim 9.8 \mathrm{kpc}$ from the line of sight to the QSO. The absorbing galaxy is unusual in that it has bright emission lines. On the basis of the optical spectrum we are unable to uniquely classify the galaxy since its emission line ratios lie in the transition region between starburst and Seyfert II type spectra.
\end{abstract}

Key words. galaxies: evolution: - galaxies: formation: - galaxies: ISM - cosmology: observations radio lines: galaxies

\section{Introduction}

Absorption lines seen in the spectra of distant quasars serve as excellent probes of intervening systems along the QSO line of sight. Of these, the highest HI column density systems, the so-called damped Lyman- $\alpha$ absorbers (DLAs), are of particular interest as they form the major repository of neutral gas at high redshifts. By studying the evolution of these DLAs, one can observationally determine the evolution of neutral gas in the universe.

The connection between the evolution of the neutral gas content and the average star formation rate in galaxies, however, remains unclear. The HI mass in DLAs at $z \sim 3$ has been found to be comparable to the stellar mass in galaxies at $z=0$, consistent with the idea that the gas in the absorbers has been converted into stars in the intervening period (Storrie-Lombardi et al. 1996). As such, this makes DLAs logical candidates for the precursors of modern-day spiral galaxies (Wolfe 1988). However, the deduced gas mass in DLAs also depends on the assumed cosmological parameters; in fact, both StorrieLombardi \& Wolfe (2000) and Peroux et al. (2001) point out that, in the currently favoured $\Omega_{\Lambda}=0.7, \Omega_{\mathrm{M}}=0.3$, $H_{0}=65 \mathrm{~km} \mathrm{~s}^{-1} \mathrm{Mpc}^{-1}$ cosmology, the estimated gas mass in DLAs at high redshift is, in fact, less than the mass in stars at $z=0$ (albeit only at the $1 \sigma$ level).

Send offprint requests to: N. Kanekar,

e-mail: nissim@ncra.tifr.res.in
Interestingly, a recent Hubble Space Telescope survey for DLAs in a sample of MgII absorbers indicates that the neutral gas content in DLAs at low redshift is comparable to that at high $z$, and is, in fact, quite consistent with a scenario in which the HI has not been converted into stars (Rao \& Turnshek 2000). The latter is, of course, a "biased" survey, since the absorbers were pre-selected on the basis of their MgII absorption; the effects of this bias are unclear. Regardless of the connection between the evolution of the neutral gas density and star formation, it remains true that the study of DLAs is currently the only observational means by which one can trace the evolution of cold neutral gas in the universe.

Besides the above, the typical size and structure of damped systems has also been an issue of much controversy. Proposed models for DLAs range from large, rapidly rotating proto-disks (e.g. Prochaska \& Wolfe 1997) to small, merging sub-galactic systems (e.g. Haehnelt et al. 1998). Locally, however, 21-cm emission studies indicate that spiral galaxies are the predominant contributors to the HI mass (Rao \& Briggs 1993); one would thus expect at least the low redshift DLAs to be primarily spiral disks. However, one of the puzzling outcomes of optical imaging of low- $z$ DLAs is that such systems appear to be associated with a wide variety of galaxy types, with only a few systems originating in luminous spirals (Le Brun et al. 1997; Turnshek et al. 2001a; Turnshek et al. 2001b; Cohen 2001; Bowen et al. 2001). 
Further, spectroscopic studies indicate that DLAs show very weak (if any) evolution in their metallicity with redshift and also do not show the expected $\alpha /$ Fe enrichment pattern expected for spiral galaxies (Pettini et al. 1999; Centurión et al. 2000). Of course, these results could well stem from selection biases in present samples of DLAs arising from, for example, issues like dust obscuration. Such issues can be addressed by detailed imaging and spectroscopic observations of individual DLAs; such studies are, however, only possible at fairly low redshifts.

Unfortunately, there are very few damped systems known at low redshifts as their identification requires UV spectra from space-based telescopes. However, all extra-galactic 21-cm absorbers for which the Lyman- $\alpha$ line has also been observed have HI column densities $N_{\mathrm{HI}}>2 \times 10^{20} \mathrm{~cm}^{-2}$, i.e. are classically damped. A detection of 21-cm absorption towards a radio-loud quasar can thus be used as a criterion for the identification of a damped system; this can then be followed up by optical/UV studies to identify the absorber.

In this paper, we describe a search for 21-cm absorption at $z=0.436$ towards the radio-loud quasar PKS 1243-072, using the Giant Metrewave Radio Telescope (GMRT). The quasar emission redshift is $z_{\mathrm{em}}=$ 1.286 (Wilkes et al. 1983). Multiple strong low ionization absorption lines (MgII $\lambda 2798$, FeII $\lambda 2600, \lambda 2587, \lambda 2383$ ) have been detected at $z_{\text {abs }}=0.436$ towards the QSO (Wright et al. 1979). The $z=0.436$ absorber was part of the MgII-selected sample searched for 21-cm absorption by Lane (2000), with the Westerbork Synthesis Radio Telescope, and was classified as a candidate $21-\mathrm{cm}$ absorber on the basis of these observations. Our fresh GMRT observations have resulted in the confirmed detection of 21-cm absorption in this system. As discussed above, this implies that the absorber fits the classical definition of a DLA. We have also carried out $R$ and $I$ band imaging studies of the system, as well as optical spectroscopy, resulting in the identification of the absorber with an $L \sim L^{\star}$ galaxy.

\section{The GMRT observations}

PKS 1243-072 was observed with the GMRT on the 1st and 2nd of January, 2001, using the standard 30 station FX correlator as the backend. A total bandwidth of $0.5 \mathrm{MHz}$ was used for the observations, sub-divided into 128 channels; this yielded a velocity resolution of $\sim 1.2 \mathrm{~km} \mathrm{~s}^{-1}$ on each run. Only 18 and 14 antennas were available on the 1 st and the 2nd respectively, due to various debugging and maintenance activity. The varying baseline coverage is, however, unimportant since PKS 1243-072 is unresolved by the longest baselines of the GMRT. Phase and bandpass calibration was carried out using the strong nearby source PKS 1127-145, while the absolute flux scale was determined using the standard calibrator 3C147. The total on-source time was $100 \mathrm{~min}$. in each run.

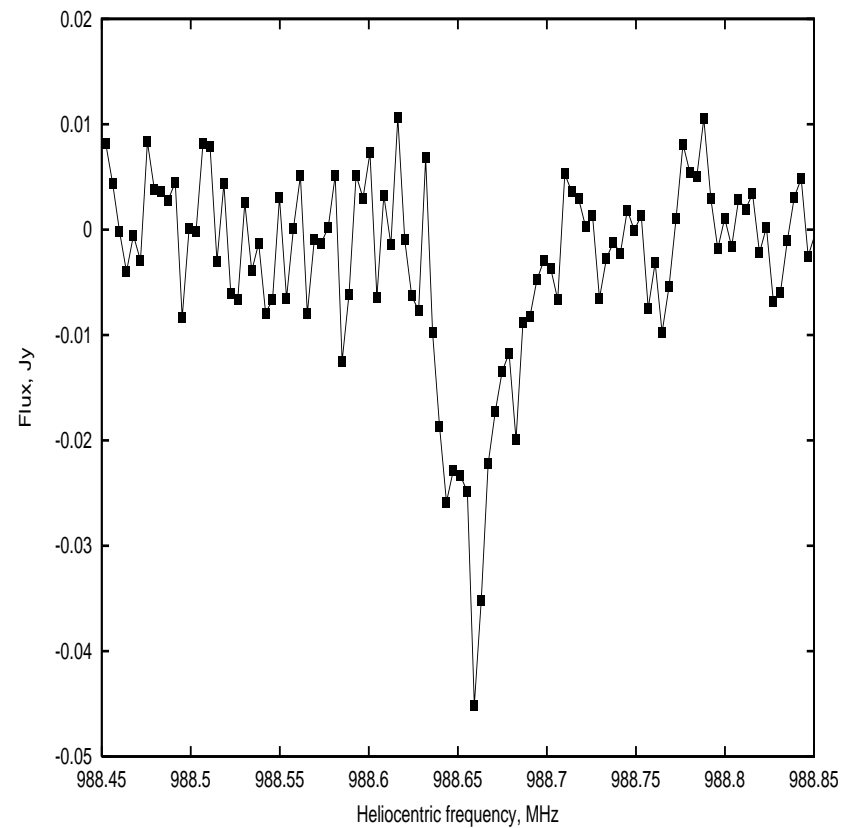

Fig. 1. GMRT 0.5 MHz HI spectrum towards PKS 1243-072. The $x$-axis is heliocentric frequency, in MHz. The spectrum has a resolution of $\sim 1.2 \mathrm{~km} \mathrm{~s}^{-1}$.

The data were converted from the telescope format to FITS and analysed in AIPS using standard procedures. Data from the two different days were analysed separately. Continuum emission was subtracted by fitting a linear polynomial to the U-V visibilities, using the AIPS task UVLIN. The continuum-subtracted data were then mapped in all channels and spectra extracted at the quasar location from the resulting three-dimensional data cube. The spectra of the two epochs were corrected to the heliocentric frame outside AIPS and then averaged together. Finally, the flux of PKS 1243-072 was measured to be $480 \mathrm{mJy}$ at both epochs. Our experience with the GMRT indicates that the flux calibration is reliable to $\sim 15 \%$, in this observing mode.

The final GMRT spectrum of the $z \sim 0.437$ system is shown in Fig. 1. The RMS noise on the spectrum is $5 \mathrm{mJy}$, per $1.2 \mathrm{~km} \mathrm{~s}^{-1}$ channel. The absorption can be seen to be spread over $\sim 20 \mathrm{~km} \mathrm{~s}^{-1}$, with a peak optical depth of $\tau=0.094$. This occurs at a heliocentric frequency of $988.659 \mathrm{MHz}$, corresponding to a redshift of $z=0.436699 \pm 0.000003$.

\section{Optical imaging and spectroscopy}

We first imaged the quasar field in the $R$ - and $I$ bands to identify candidates responsible for the absorption lines seen at 21-cm (this paper) and optical (Wright et al. 1979). As discussed below, the optical imaging revealed the presence of a bright galaxy close to the line of sight to the QSO. Subsequent long slit spectroscopy of the neighbour showed that this system is indeed at $z=0.437$ and hence likely to be the 21 -cm absorber. 


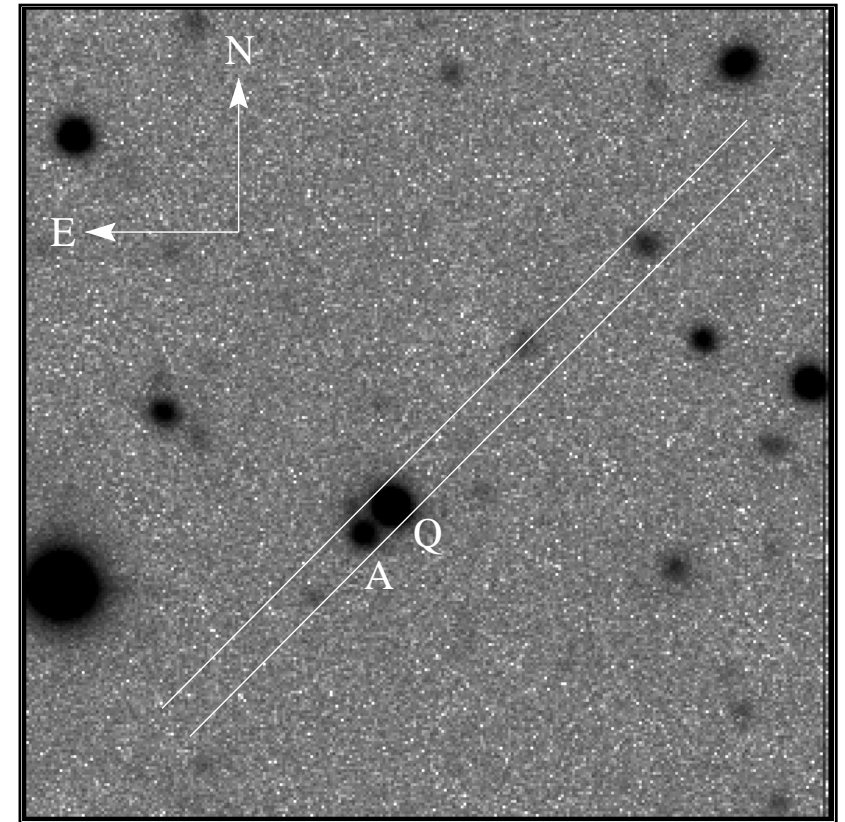

Fig. 2. An $R$-band image of the field of the quasar PKS 1243-072. The grey-scale plot is 45 arcsec on each side with the orientation as marked. The two lines represent the long-slit $(\mathrm{PA}=-44.9 \mathrm{deg})$ used to obtain the spectra of the quasar $(\mathrm{Q})$ and the candidate absorber (A). The 2 objects are separated by 2.2 arcsec on the sky.

\subsection{Optical imaging}

The quasar field was imaged using the EFOSC2 instrument on the ESO 3.6m telescope at La Silla during the night of 29th January, 2001. The Bessel $R$-band image was obtained on CCD \#40 in the unbinned mode (0.157 arcsec/pix, $2060 \times 2060$ pixels $\equiv 5.4 \times 5.4$ arcmin field) . We obtained a total of ten dithered images of $450 \mathrm{~s}$ each. The calibration observations included twilight sky flats and observations of the standard field RU152 (Landolt 1992). We used the science frames to construct and subtract the fringe pattern from the frames. The atmospheric extinction and the CCD colour term were calculated using observations of standard fields taken at several airmasses and in other bands. We expect the photometry to be accurate to about $5 \%$ and use a conservative error of $0.1 \mathrm{mag}$. The images were reduced, calibrated, registered and co-added in a standard manner using IRAF. The final image obtained had a zero-point of $R=32.601$ with an rms of 10.5 counts/pix.

Figure 2 shows a greyscale plot of a $45 \times 45$ arcsec section of the field around the quasar. The quasar $(\mathrm{Q})$ and a rather bright neighbour (A), separated by 2.2 arcsec, are visible, close to (and south of) the centre of the field. The quasar magnitude was measured to be $R=19.55$ while that of its neighbour was found to be $R=21.27$.

We also obtained an image of the field using the Magellan $6.5 \mathrm{~m}$ telescope at the Las Campanas Observatory. The Harris $I$-band image was obtained on a direct $\mathrm{CCD}$ camera in the unbinned mode $(0.069 \mathrm{arcsec} / \mathrm{pix}$, $2048 \times 2048$ pixels $\equiv 2.36 \times 2.36$ arcmin field) . We

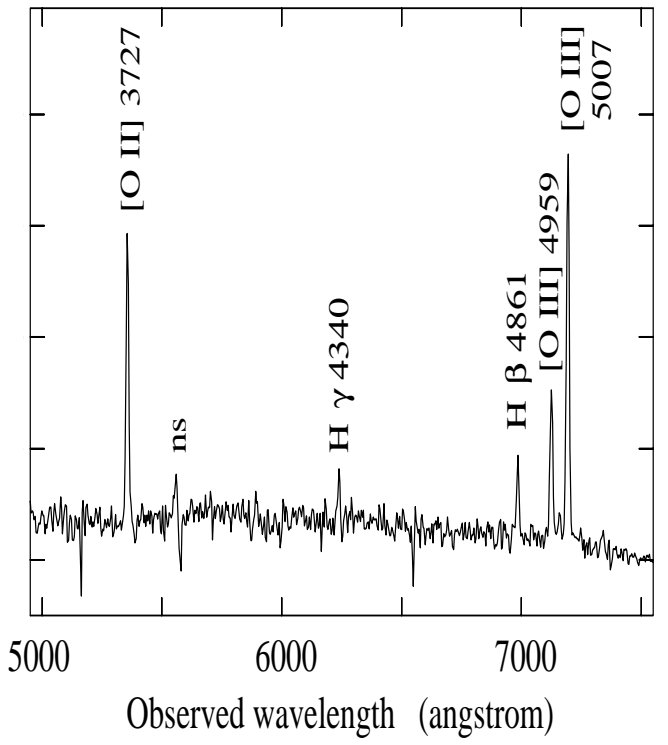

Fig. 3. An optical spectrum of the candidate absorber (object A on Fig. 2 in the field of the quasar PKS 1243-072. The intensity scale ( $y$-axis) is linear but in arbitrary units. The labels corresponding to each emission line identify the species and the rest wavelength (ns: night sky feature). The average redshift derived from the emission lines is $z=0.437$.

obtained 4 exposures of $600 \mathrm{~s}$ each; the images were reduced in the same manner as before. However, it may be noted that, due to technical problems, we could not obtain a sky/dome flat and had to use the dark-sky flat to flat-field the image. The fringes on the images were hence divided out rather than subtracted which resulted in an additional error of a few per cent in the photometry across the frames. However, the total photometric error is again about $0.1 \mathrm{mag}$.

We measured magnitudes of $I=19.41$ for the quasar and $I=21.27$ for the neighbour.

\subsection{Optical spectroscopy}

The optical long-slit spectrum was obtained using the EFOSC2 instrument on the ESO $3.6 \mathrm{~m}$ telescope during the night of 19th March, 2001. Grism \#11 (300 l/mm, 3300-7520 A) was used with CCD \#40 (binned $2 \times 2$ pixel size 0.314 arcsec) to obtain a spectrum with a resolution of $4.1 \AA /$ pixel. The standard calibration included He-Ar arc lamp exposures for wavelength calibration and dome flat exposures to eliminate the pixel-to-pixel gain variation. A $2^{\prime \prime}$ slit was oriented so as to include both the quasar and the candidate absorber (see Fig. 2). The observations were split into 3 runs of $1800 \mathrm{~s}$ each, to eliminate cosmic rays. The data were reduced in a standard manner using the IRAF package.

The spectrum of the candidate absorber is shown in Fig. 3. It should be noted that the night was not photometric and no effort has been made to correct the spectrum for the CCD spectral response using a standard star observation. However, we used a previous determination of 
the CCD spectral response to calibrate the spectra with the limited aim of estimating spectral line ratios. Thus, while the absolute calibration may be significantly wrong, the line ratios should be good to $\sim 15 \%$. We obtained a mean redshift of $z=0.4370 \pm 0.0001$ using the 5 emission lines identified in the spectrum. The close correspondence between this emission line redshift and the redshifts of the absorption lines seen in the quasar spectra imply that object $\mathrm{A}$ is indeed likely to be the galaxy responsible for the absorption line system.

\section{Discussion}

\subsection{HI column density}

The $21 \mathrm{~cm}$ optical depth, $\tau_{21}$, of an optically thin, homogeneous cloud is related to the column density of the absorbing gas $N_{\mathrm{HI}}$ and the spin temperature $T_{\mathrm{s}}$ by the expression (e.g. Rohlfs 1986)

$N_{\mathrm{HI}}=\frac{1.823 \times 10^{18} T_{\mathrm{s}}}{f} \int \tau_{21} \mathrm{~d} V$,

where $f$ is the covering factor of the absorber. In the above equation, $N_{\mathrm{HI}}$ is in $\mathrm{cm}^{-2}, T_{\mathrm{s}}$ in $\mathrm{K}$ and $\mathrm{d} V$ in $\mathrm{km} \mathrm{s}^{-1}$. For a multi-phase absorber the spin temperature derived using the above expression is the column density weighted harmonic mean of the spin temperatures of the individual phases.

In the case of PKS 1243-072, VLBA maps at 2.3 and $8.4 \mathrm{GHz}$ (see the Radio Reference Frame Image Database of the United States Naval Observatory) show that the entire flux is contained within $\sim 20$ milliarcseconds. The extremely small size of the background source makes it highly likely that the covering factor $f$ is close to unity. This implies an $\mathrm{HI}$ column density $N_{\mathrm{HI}}=3 \times$ $10^{20}\left(T_{\mathrm{s}} / 200 \mathrm{~K}\right) \mathrm{cm}^{-2}$; given that all known DLAs have $T_{\mathrm{s}} \geq 150 \mathrm{~K}$ (Chengalur \& Kanekar 2000), it is quite likely that the absorber has $N_{\mathrm{HI}}>2 \times 10^{20} \mathrm{~cm}^{-2}$ (i.e. fits the classical definition of a DLA) even if it has a low spin temperature. Of course, a spin temperature $\gtrsim 1000 \mathrm{~K}$, more typical of DLAs (Chengalur \& Kanekar 2000), would imply a much higher column density, $N_{\mathrm{HI}} \gtrsim 10^{21} \mathrm{~cm}^{-2}$. However, the high luminosity of the absorber makes it likely that its spin temperature is low; this is discussed in more detail in the next section. Observations of the Lyman- $\alpha$ line using the Hubble Space Telescope (HST) will provide a direct estimate of the column density and thus, of the spin temperature.

\subsection{The nature of the absorber}

The rather strong optical emission lines seen in the absorber are rather unusual. Further, the absorber is only slightly more extended than the quasar image $\left(Q_{\mathrm{FWHM}}=\right.$ 0.96 arcsec and $A_{\mathrm{FWHM}}=1.08$ arcsec for the QSO and object A on the $R$-band image, and 0.71 and 0.86 arcsec respectively, on the $I$-band image). In fact, object A appears more point-like than most of the other galaxies in the field.
The emission lines could be either due to star-burst activity or due to the presence of an active galactic nucleus (AGN). It is possible to distinguish between these two scenarios on the basis of the ratios of the strengths of certain emission lines. Dessauges-Zavadsky et al. (2001; hereafter DZ01) present template spectra of different emission line galaxies and also discuss their own and earlier classification schemes (Rola et al. 1997; Tresse et al. 1996). A visual inspection of the template spectra clearly shows that the object A is either a Seyfert II system or a starburst galaxy. Next, the spectrum of object A has the following line ratios

$[\mathrm{OIII}] \lambda 5007 / \mathrm{H} \beta \sim 6.2$

$[\mathrm{OII}] \lambda 3727 / \mathrm{H} \beta \sim 3$

\section{[OIII] $\lambda 4959 / \mathrm{H} \beta \sim 2.2$}

which can be used as diagnostics for the purpose of classification (Rola et al. 1997; Tresse et al. 1996). A comparison of these values with Fig. 7 of DZ01 shows that object $\mathrm{A}$ falls in between the range of ratios obtained in typical HII galaxies and Seyfert II type systems. DZ01 also presented a new diagnostic, based on a comparison between the quantity $R_{23} \equiv([\mathrm{OII}] \lambda 3727+[\mathrm{OIII}] \lambda 4959+$ $[\mathrm{OIII}] \lambda 5007) / \mathrm{H}_{\beta}$ and the ratio $[\mathrm{OI}] \lambda 6300 / \mathrm{H} \alpha$. We unfortunately do not have a measurement of either $[\mathrm{OI}] \lambda 6300$ or $\mathrm{H} \alpha$ in object $\mathrm{A}$; however, we do estimate $\log \left[R_{23}\right]=1.06$ for this system. DZ01 noted that $87 \%$ of all Seyfert II galaxies had $\log \left[R_{23}\right]>1.1$ and, an inspection of Fig. 8 in DZ01 shows that all Seyfert II systems lie above $\log \left[R_{23}\right]=1.05$. Again, object $\mathrm{A}$ lies close to the border separating Seyfert II and starburst galaxies. We note that all the above diagnostics tend to place object A marginally amongst the starburst systems. Given the lack of a measured $[\mathrm{OI}] / \mathrm{H} \alpha$ ratio and the problem with spectrophotmetric calibration mentioned earlier, we are unable to conclusively distinguish between the two possibilities. We plan to carry out observations of the $[\mathrm{OI}] \lambda 6300$ and $\mathrm{H} \alpha$ lines from this system, which should be useful in resolving the issue.

The 2.2 arcsec separation between the quasar and the absorber corresponds to a linear separation of $9.8 \mathrm{kpc}$ between their lines of sight at the redshift of the absorber (assuming a flat FRW Universe, with $H_{0}=$ $\left.75 \mathrm{~km} \mathrm{~s}^{-1} \mathrm{Mpc}^{-1}\right)$. Although we cannot rule out the possibility that the absorbing galaxy is not object $\mathrm{A}$, but some fainter companion galaxy, the small projected separation between object $\mathrm{A}$ and the QSO makes it likely that the absorption arises in object $\mathrm{A}$ itself. We note that there is a faint object $1^{\prime \prime}$ north of $\mathrm{A}$ and $2^{\prime \prime}$ east of the quasar, barely visible in Fig. 2. This system is about 2 magnitudes fainter than object A and considerably more diffuse. It is unclear whether this object is in the vicinity of the QSO or a companion to object $\mathrm{A}$ or, indeed, an interloper not associated with either system. 
Our optical photometry shows that object A has $L \sim$ $L^{\star}$. This is consistent with the results of Rao \& Briggs (1993) who used a survey of the HI content of $z=0$ optically bright galaxies, to conclude that the cross section for DLA absorption peaks at this luminosity. Note, however, that Rosenberg \& Schneider (2001) argue that a substantial contribution to the DLA cross-section is provided by optically faint galaxies, based on a blind 21-cm survey at $z=0$. The latter is also consistent with optical searches for the counterparts of low redshift DLAs, which have shown that the absorbers arise in galaxies with a wide range of luminosities.

Chengalur \& Kanekar (2000) found that low spin temperatures $\left(T_{\mathrm{s}} \lesssim 300 \mathrm{~K}\right)$ were obtained in the few cases where the absorber was identified to be a spiral galaxy; such temperatures are typical of the Milky Way and local spirals (see also Kanekar \& Chengalur 2001). However, the majority of DLAs were found to have far higher spin temperatures, $T_{\mathrm{s}} \gtrsim 1000 \mathrm{~K}$. Higher $T_{\mathrm{s}}$ values are to be expected in smaller systems like dwarf galaxies, whose low metallicities and pressures are not conducive to the formation of the cold phase of HI (Wolfire et al. 1995); such systems hence have a higher fraction of warm gas as compared to normal spirals, and therefore, a high spin temperature. On the other hand, bright galaxies tend to have high masses, and hence both higher metallicities and central pressures, contributing to the formation of the cold phase of neutral hydrogen. The high luminosity of object $A$ thus indicates that it is likely to have a low spin temperature $(\$ 300 \mathrm{~K})$ and hence, a relatively low column density $N_{\mathrm{HI}} \lesssim 5 \times 10^{20} \mathrm{~cm}^{-2}$. It would be interesting to test this conjecture by means of HST observations in the Lyman- $\alpha$ line, as well as to directly determine the metallicity of the absorber through high resolution absorption studies. In a subsequent paper, we plan to compare the metallicity as computed from such a high resolution absorption spectrum to the metallicity measured from the emission lines.

Acknowledgements. The radio observations presented in this paper would not have been possible without the many years of dedicated effort put in by the GMRT staff to build the telescope. The GMRT is run by the National Centre for Radio Astrophysics of the Tata Institute of Fundamental Research. The optical observations were carried out using European Southern Observatory facilities at La Silla Observatory, Chile. This research has made use of the United States Naval Observatory (USNO) Radio Reference Frame Image Database (RRFID). This research has made use of the NASA/IPAC Extragalactic Database (NED) which is operated by the Jet Propulsion Laboratory, California Institute of Technology, under contract with the National Aeronautics and Space Administration.
Note added in proof: $21 \mathrm{~cm}$ absorption from 1243-072 has recently been independently detected by Lane et al. (2001) using the WSRT.

\section{References}

Bowen, D. V., Tripp, T. M., \& Jenkins, E. B. 2001, AJ, 121, 1456

Centurión, M., Bonifacio, P., Molaro, P., \& Vladilo, G. 2000, ApJ, 536, 540

Cohen, J. G. 2001, AJ, 121, 1275

Chengalur, J. N., \& Kanekar, N. 2000, MNRAS, 318, 303

Dessauges-Zavadsky, M., Pinado, M., Maeder, A., \& Kunth, D. 2000, A\&A, 335, 89 (DZ01)

Haehnelt, M. G., Steinmetz, M., \& Rauch, M. 1998, ApJ, 495, 64

Kanekar, N., \& Chengalur, J. N. 2001, A\&A, 369, 42

Landolt, A. U. 1992, AJ, 104, 340

Lane, W. 2000, Ph.D. Thesis, Univ. of Groningen

Lane, W., \& Briggs, F. H. 2001, ApJ, 561, L27

Le Brun, V., Bergeron, J., Boissé, P., \& Deharveng, J.-M. 1997, A\&A, 321, 733

Peroux, C., McMahon, R. G., Storrie-Lombardi, L. J., \& Irwin, M. J. 2001, MNRAS, submitted [astro-ph/0107045]

Pettini, M., Ellison, S. L., Steidel, C. C., \& Bowen, D. V. 1999, ApJ, 510, 576

Prochaska, J. X., \& Wolfe, A. M. 1997, ApJ, 487, 73

Rao, S. M., \& Briggs, F. H. 1993, ApJ, 419, 515

Rao, S. M., \& Turnshek, D. A. 2000, ApJS, 130, 1

Rohlfs, K. 1986, Tools of Radio Astronomy (Springer-Verlag, Berlin Heidelberg)

Rola, C. S., Terlevich, E., \& Terlevich, R. J. 1997, MNRAS, 289, 419

Rosenberg, J. L., \& Schneider, S. E. 2001, to appear in Extragalactic Gas at Low Redshift [astro-ph/0107495]

Storrie-Lombardi, L. J., McMahon, R. G., \& Irwin, M. J. 1996, MNRAS, 283, L79

Storrie-Lombardi, L., \& Wolfe, A. M. 2000, ApJ, 543, 552

Tresse, L., Rola, C. S., Hammer, F., et al. 1996, MNRAS 281, 847

Turnshek, D. A., Rao, S. M., Lane, W., et al. 2001a, in Gas and Galaxy Evolution, ed. J. E. Hibbard, M. P. Rupen, \& J. H. van Gorkom, ASP Conf. Ser., 240, 61

Turnshek, D. A., Rao, S. M., Nestor, D., et al. 2001b, ApJ, 553,288

Wilkes, B. J., Wright, A. E., Jauncey, D. L., \& Peterson, B. A. 1983, PASA, 5, 2

Wolfe, A. M. 1988, in QSO Absorption Lines: Probing the Universe, ed. J. C. Blades et al. (Cambridge University Press)

Wolfire, M. G., Hollenbach, D., McKee, C. F., Tielens, A. G. G. M., \& Bakes, E. L. O. 1995, ApJ, 443, 152

Wright, A. E., Peterson, B. A., Jauncey, D. L., \& Condon, J. J. 1979, ApJ, 229, 73 